\title{
Development of a new lines of sight analyzer while playing sport
}

\author{
Shinya Mochiduki ${ }^{1}$, Miyuki Suganuma ${ }^{1}$, Gaito Shoji ${ }^{2}$, Mitsuho Yamada ${ }^{1}$ \\ ${ }^{1}$ Graduate of school of Information and Telecommunication Engineering, Tokai University, 108-8619, Japan \\ ${ }^{2}$ School of Information and Telecommunication Engineering, Tokai University, 108-8619, Japan
}

\begin{tabular}{l} 
A R T I C L E I \\
\hline Article history: \\
Received: 20 Decen \\
Accepted: 20 Jan \\
Online: 28 Januar \\
\hline Keywords: \\
Eye movement \\
head movement \\
athlete \\
baseball \\
lines of sight
\end{tabular}

\section{Introduction}

Various efforts to improve the movement ability and the play of top athletes by objectively estimating their competitive ability will be undertaken before the Tokyo Olympic Games in 2020.

Sports research for the skilled players and the unskilled players have been researched a lot [1,2]. When working to improve an athlete's performance, the movement of the athlete's eyes is a particular area of focus. Skilled athletes obtain important information through peculiar visual search patterns, not disorderly eye movement [3]. The measurements in the actual athletic sites are also conducted [4]. For example, it is often said that a player should not take his or her eyes off the ball in ball games, but the speed of the ball is too fast for anyone to follow with smooth pursuit eye movement, especially in tennis. Tennis players predict the landing point of a tennis ball and move his/her eyes to that point [5]. On the other hand, in an experiment that focused on the

${ }^{*}$ Corresponding Author: Shinya Mochiduki, Graduate of school of Information and Telecommunication Engineering Tokai University, +810334411171, s.mochiduki@star.tokai-u.jp accuracy of smooth pursuit eye movement, the position error in pursuing a target moving at $70 \mathrm{deg} / \mathrm{sec}$ was found to be $6 \sim 8$ degrees for a general university student, although this error is less than 2 degrees for university students who are table tennis players [6].

The convenonal measurement of the eye movement of athletes has been performed using a system in which the athlete wears eyemovement-measuring equipment while playing [5] or a system in which the athlete's head is held stationary on a head-chin rest while he or she views a video showing action from his or her sport [5]. Movement of the line of sight is not only determined by eye movement; head movement is also important. When eyemovement-measuring equipment is worn by an athlete and the movement of the eyes is measured, the camera attached to the athlete's forehead moves with the player's intense movement, and analysis of the video after the experiment becomes difficult. Though head movement is indispensable for acquiring information from the wide viewing angle of human vision, head movement itself has not often been measured. Equipment with which eye movement and head movement can be measured at the same time 
was developed [7], and a new method was proposed for the analysis of the coordination of head and eye movement in sport. One objective of this research is to actually measure the lines of sight of an athlete using equipment worn by the athlete, but in the present research, an athlete in a laboratory observes video of a game which was recorded using a $4 \mathrm{~K}$ camera in order to assess the method.

\section{Experimental Method}

An experiment was performed involving baseball as the sport to be studied. We fixed a wearable camera, a Panasonic HX-A500 [8] on a tripod behind the catcher and behind the runner and recorded a video. A 55-inch screen (Regza 55X3, Toshiba, Tokyo, Japan) on which a $4 \mathrm{~K}$ picture can be displayed was used, and an experimental picture was output. The distance between the display and the subject was set to $51 \mathrm{~cm}$, which was 0.75 times the screen height, to reproduce the view of the catcher, and the viewing angle was 102 degrees. For the eye-movement measurements, the system used a corneal reflex eye-tracking recorder (EMR-8B, Nac Image Technology, Tokyo, Japan) [9]. To measure head movement, a Patriot magnetic position detecting system (Polhemus, Colchester, VT, USA) was used, which can measure motion at 6 degrees of freedom (DOF) [10]. The sensors of the magnetic positiondetecting system and the eye-tracking recorder were attached to the subject's cap. The source coil was fixed on a wooden pillar installed at the subject's right-hand side. We decided not to use ironwork around the subject and during the measurement to avoid any obstruction of magnetism. The data from the eye-movement and head-movement measuring systems were unified and changed into gaze movement on a PC [7]. The equipment used in the experiment is shown in Table 1.

Table 1. Equipment used in the experiment

\begin{tabular}{|c|c|}
\hline Video & $\begin{array}{c}4 \mathrm{~K} \text { Wearable camera (Panasonic HX- } \\
\text { A500)[8] }\end{array}$ \\
\hline $\begin{array}{c}\text { Eye movement } \\
\text { measurement }\end{array}$ & $\begin{array}{c}\text { EMR-8B, Nac Image Technology, Tokyo, } \\
\text { Japan [9] }\end{array}$ \\
\hline $\begin{array}{c}\text { Head movement } \\
\text { measurement }\end{array}$ & $\begin{array}{c}\text { Patriot magnetic position detecting system } \\
\text { (Polhemus, Colchester, VT, USA)[10] }\end{array}$ \\
\hline
\end{tabular}

The system configuration is shown in Figure 1 . The $4 \mathrm{~K}$ video was played on a personal computer and displayed on a $4 \mathrm{~K}$ monitor (REGZA 55X3 55, Toshiba, Tokyo). For the composition of the $4 \mathrm{~K}$ video that was viewed by the subject and the gaze movement, the system uses ATEM Production Studio 4K Switchers [11] (Blackmagic Design, Fremont, CA, USA). The $4 \mathrm{~K}$ video is connected to the ATEM Production Switchers through an HDMI output, and a superimposed image representing the movement of the subject's gaze is displayed on the experimenter's display and recorded in the solid-state drive of a non-compression video recorder (HyperDeck Shuttle; Blackmagic Design, Fremont, CA, USA). In this way, it is possible to superimpose a player's line of sight, which is a combination of head movement and eye movement, on the wide-view picture, which corresponds to the view of the player and is recorded from behind the player by a fixed camera on a tripod. The subjects were three baseball players at our university; all were males ranging from 21 22 years old; two usually played as catchers and one usually played as an outfielder.

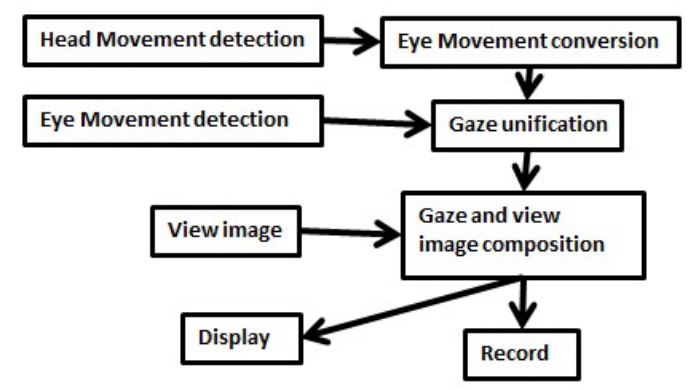

Figure 1. System configuration

\section{Experimental Procedure}

We took video of an intra-squad game of a club that plays "a Japanese version of baseball played with a hard rubber ball", Highrings, which was made up of students from our university. The video was recorded from behind the catcher, from beside the first baseman, and from beside the third baseman using a $4 \mathrm{~K}$ camera fixed on a tripod so as not to interfere with the game. The video was edited to a length of around five minutes and separated into three scenes, a scene shot from behind the catcher, a scene shot from the first baseman's side, and a scene shot from the third baseman's side, and a still image of a fixation point on the center of the screen was inserted between scenes to confirm the eye movement accuracy. Before the experiment, the subjects were instructed to watch the video as if actually playing in the game, for example, as a catcher for the scene shot from behind the catcher, and as a runner for the scenes shot from beside the first baseman and third baseman. We had the subjects watch the video wearing eye-movement and head-movement sensors without fixing the head.

\section{Experimental Result}

Here we propose a new method of analysis of eye and head coordination during a sports competition involving the simultaneous measurement of eye movement and head movement. An image of the player's view moves according to the head movement by means of a conventional system in which eye movement is superimposed on a view image at the forehead part of the player. Figure 2 shows an example of the view image moving according to the head movement. On the other hand, the line of sight (head movement + eye movement) is superimposed on the fixed image taken from behind the player using the newly developed system. Since the lines of sight of the player are superimposed on the fixed camera image taken from behind the player, the lines of sight are reproduced on the fixed image instead of the view image, which moves according to the head movement as shown in Figure 3.

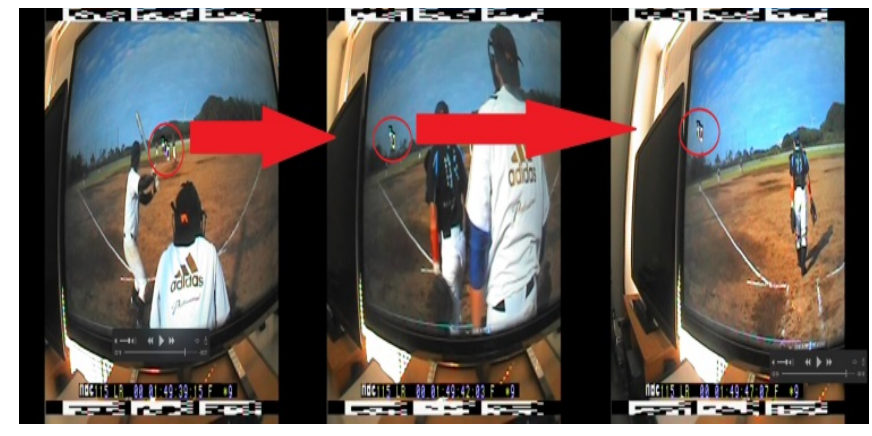

Figure 2. Example of eye movement by conventional experimental method 


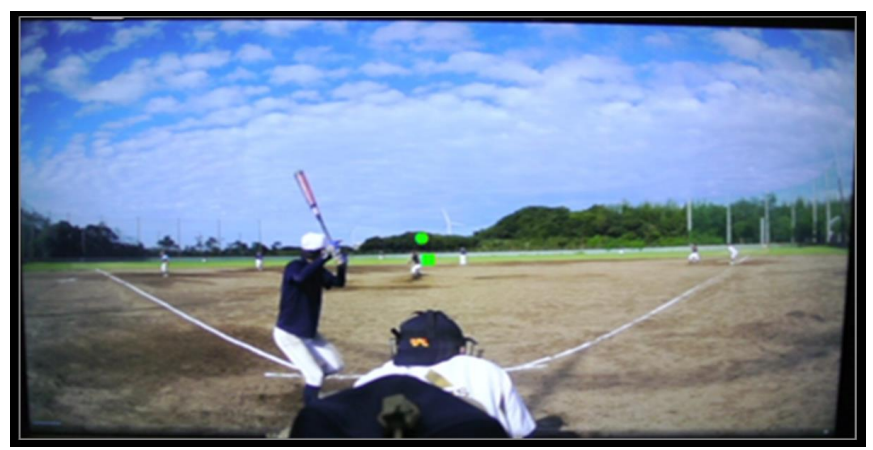

Figure 3. Example of line of sight (head movement + eye movement) determined by our newly proposed method

Here, we discuss the results obtained when the subjects viewed the video recorded from behind the catcher. This video assumed that a catcher watches a first-base runner cautiously because the catcher is responsible for catching any pitch thrown by the pitcher that bounces near home plate to keep the runner from advancing. The runner's focus is on the next base because there is a possibility that the ball could get past the catcher when the pitch bounces near home plate. Therefore, when a pitcher throws a pitch that bounces near home plate, the catcher catches it or blocks it with his or her body, then confirms whether or not the runner is running to the next base.

Figure 4 indicates the result when the lines of sight of subjects A, B and C are all included. First, the lines of sight of subjects A and $\mathrm{C}$, who have played the position of catcher, were on home plate where a ball thrown by the pitcher bounced, and they then moved their gaze to the first-base runner. The line of sight of subject B, who has not played the catcher position, always pursued the ball. The part enclosed in a blue circle in Figure 4 shows that the lines of sight of subjects A and C both went toward a place where the line of sight of subject B did not go.

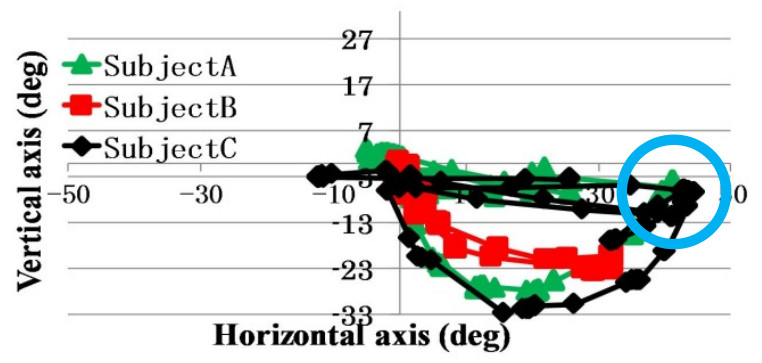

Figure 4. Three subjects' lines of sight (eye movement + head movement) as a catcher

Two runners on first base and second base, two outs, and the fourth player is the batter in the situation in the second experiment. The first-base runner and second-base runner start as soon as the batter hits the ball. As for this video, the batter hits an extra-base hit past the outfielders to the left, and the first-base runner and second-base runner reach home plate. The batter who hit the ball is advancing to second base. Figures 5 and 6 show the Eye movement of subjects A and B, respectively. Figure 7 indicates the amount of head movement of both subjects, and Figure 8 shows the result when the lines of sight of subjects $\mathrm{A}$ and $\mathrm{B}$ are combined. Regarding the lines of sight of subject A, who had experience as a catcher, movement to follow the ball accounted for most of his movement, but lines of sight to watch the movement of the batter who ran were also seen. The result for subject B, who had no experience as a catcher, showed eye movement that mostly followed the ball. In subject A, there was less head movement than in subject $\mathrm{B}$ when we compared the amounts of head movement from Figure 7. From Figure 8, in which the movement of the lines of sight of subjects A and B is shown simultaneously, only subject $\mathrm{A}$ is looking around second base (a light blue circle in the figure), which the batter eventually reached.

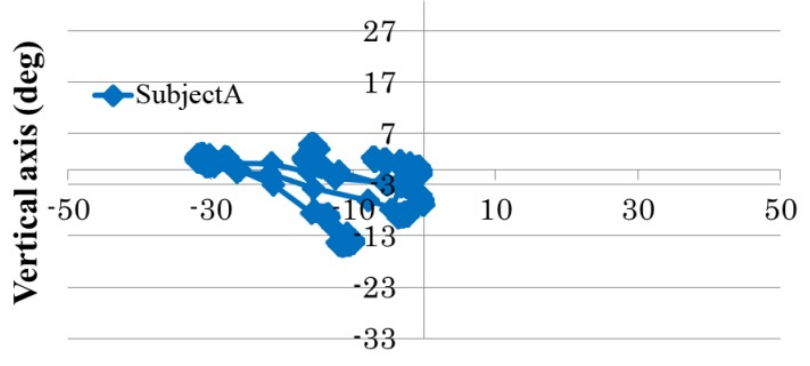

Horizontal axis (deg)

Figure 5. Subject A's Eye movement as a catcher

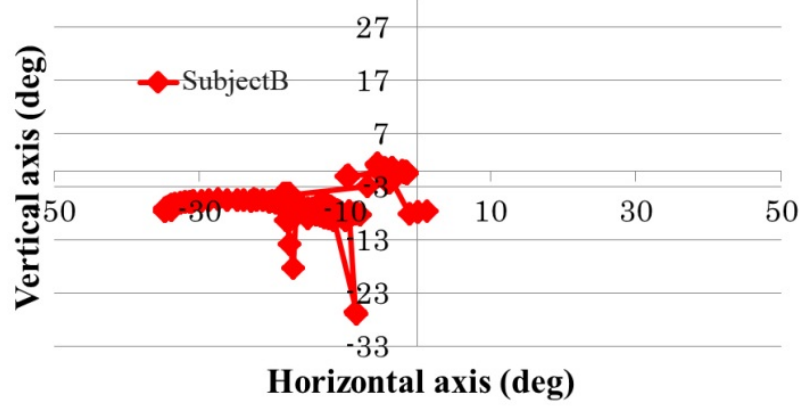

Figure 6. Subject B's eye movement as a catcher

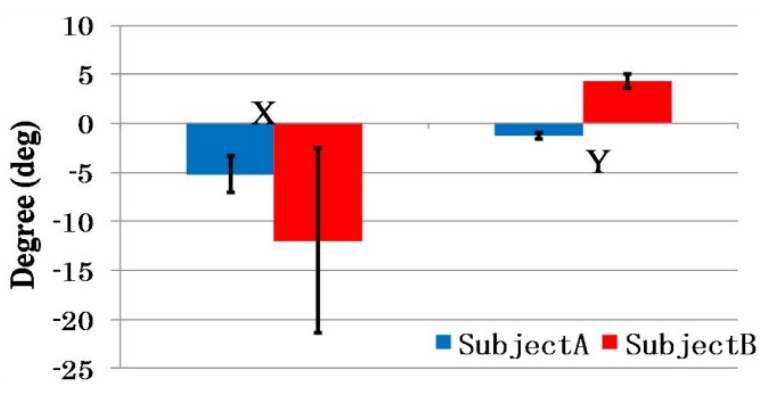

Figure 7. Amount of movement of the head

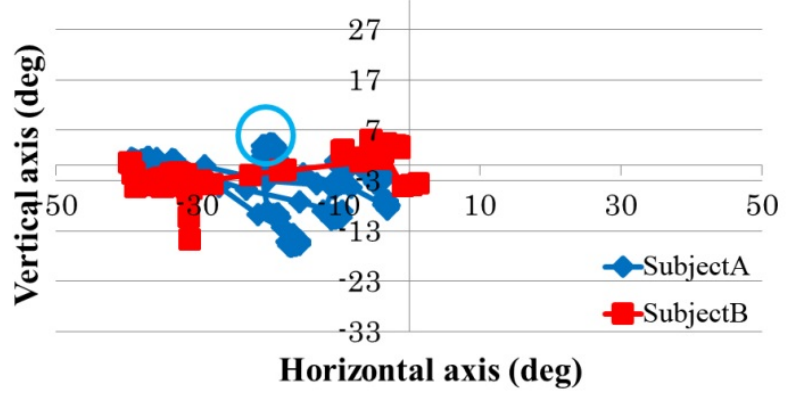

Figure 8 . Two subjects' lines of sight (eye movement + head movement) as a catcher 


\section{Discussion}

From the first finding, a difference was seen in the movement of lines of sight in a subject who had no experience as a catcher compared with a subject who was an experienced catcher. The subject who was an experienced catcher showed a tendency to mind the runner while following the ball while the subject without experience as a catcher always followed the ball. It was suggested that the viewpoint of the game was different for a player in a defensive position based on the movement of the lines of sight.

The second finding suggested that a subject who was an experienced catcher monitored a wide field of view using only eye movement and with little head movement, because the moving area of the lines of sight was not very much different between the subject who was an experienced catcher and the subject who was not, even though the subject who was an experienced catcher showed very little head movement. Although it is said that highlevel athletes move their heads well and monitor a wide field of vision, it is also said that athletes running marathons who become tired show slight head movement. In other words, runners who are running stably run without their heads moving, even slightly. A tendency to monitor a wide field of vision closely by eye movement was seen in the subjects who were experienced catchers and who did not move their heads significantly in this experiment. While the head movement was small in these subjects, they showed more eye movement by saccade than an inexperienced person.

Here we compare the result in the past research and the present experiment. Gorman et al. and Land et al. have reported that experienced players predictively look ahead their lines of sight to the next destination $[12,13]$. In our experimental results, the experienced players predict the runners' movement and they moved their lines of sight to the first base. The result that the no experienced player focuses his attention exclusively on the ball is also supports the result of Williams et al. [14]. From these results, we considered that there are no differences between the past researches result and the present experiment by proposed method.

Urgesi et al. has reported that actual player predict the next movement better than the watching learner in the volleyball learning experiment between two groups: learn by playing and learn by watching others' play [15]. This result also supports our result because we could see the differences between the experienced catcher and the unexperienced catcher in our present experiment results.

\section{Summary}

Given that the Olympics will be held in Tokyo in 2020, the training of athletes using technology has begun to attract a great deal of attention. In Japan, an effort to improve athletes' performance is being led by a national scientific center. In order to improve the athletic capabilities of elite athletes by evaluating their skills objectively, we have studied their eye movement and head movement. Since the field of view moves according to the head movement of the athlete, which was a problem for the conventional method of measuring eye movement, we proposed a new method of analysis of lines of sight which could record the head movement during competition and make it easier to analyze by superimposing the lines of sight on an externally recorded fixed image. The ultimate objective is to attach the equipment to the athlete and measure the lines of sight during competition, but in this study we recorded a video during competition before the experiment was conducted to confirm the effectiveness of the method, and we had athletes view the video in a laboratory.

We recorded our video during a baseball game. First, we compared a video in which only eye movement was measured and the field-of-view image moved according to the head movement of the player with another video in which the head movement and eye movement were both measured and the image did not move in spite of the player's head movement. The experiment showed differences between the eye movement of an experienced person and that of an inexperienced person, particularly between those who had experience playing the position of catcher and those who did not. We would like to measure the line of sight of an athlete actually playing a game by attaching the head and eye movement sensors to an athlete in the future.

\section{Acknowledgment}

We would like to express our sincere gratitude to the "High rings" student baseball team at our university for kindly allowing us to take movies of them playing.

\section{References}

[1] S.Mori, T.Shimada, "Expert anticipation from deceptive action,Attention" Perception, \& Psychophysics,May 2013, 75(4), 751-770, 2013.

[2] S.Mori, "Spatiotemporal occlusion of biological motion reveals anticipatory processes of expert players" JSPS Grant Kickoff Workshop "Experimental study on perception-action systems in real complex environments", 2009.

[3] T. Kato, "Skill of the expert who looked from visual science, the latest sports psychology trace and prospects" Sport psychology,163-174, 2004.

[4] A.M.Williams, K.Davids, J.G.Williams. Visual perception and action in sport, E \& FN Spon. 1999.

[5] M. Yamada, T. Fukuda, "A New Sight-line Displacements Analyser and Its Application to TV Program Production SMPTE Journal” J SMPTE., 99(1), 16-26, 1990.

[6] M. Saito, Y. Ogata, M. Yamada, "Development of eye-movement analysis equipment for clarifying the visual characteristics of moving images" The First International Workshop on Image Media Quality and its Application,135-140,2007.

[7] H. Takahira, K. Kikuchi, M. Yamada, "A System for Measuring Gaze Movement and Hand Movement Simultaneously for Hand-Held Devices" IEICE Trans. Commun, 98(1), 51-61, 2015.

[8] http://panasonic.jp/wearable/a500/in Japanese

[9] http://www.nacinc.com/datasheets/archive/EMR9-Data-Sheet-June-09.pdf ,latest version of EMR8-B

[10] http://polhemus.com/motion-tracking/all-trackers/patriot/

[11] https://www.blackmagicdesign.com/jp/products/atem/techspecs/W-APS-04

[12] A. D. Gorman, B. Abernethy, D. Farrow, “,Investigating the anticipatory nature of pattern perception in sport.”, Mem Cognit, 39(5), 894-901, 2011. 
[13] M. F. Land, P. McLeod, "From eye movements to actions: how batsmen hit the ball" Nature Neuroscience 3, 1340 - 1345, 2000.

[14] A. M. Williams, K. Davids, L. Burwitz, J. G. Williams, "Visual search strategies in experienced and inexperienced soccer players" Research Quarterly for Exercise and Sport, 65(2), 127-135, 1994.

[15] C. Urgesi, M. M. Savonitto, F. Fabbro, S. M. Aglioti, "Long- and short-term plastic modeling of action prediction abilities in volleyball" Psychol Res., 76(4), 542-560, 2012. 